\title{
SOME SEMIGROUPS ON A MANIFOLD WITH BOUNDARY'
}

\author{
T. H. MCH. HANSON
}

Abstract. In this paper, $S$ is an abelian semigroup on an n-dimensional simply connected manifold with boundary whose interior is a dense, simply connected, connected Lie group. We also assume there is a vector semigroup $V_{k}^{-}$in $S$ such that the interior of $S$ misses the boundary of $V_{k}^{-}$, and such that $\left(S-G L_{k}\right) / V_{k}$ is a group. It is shown that if $k=n$, then $S$ is iseomorphic to $V_{n}^{-}$, and if $k=1,2$, or $n-1$, then $S$ is iseomorphic to $V_{n-k} \times V_{k}^{-}$.

Introduction. In this paper we employ the language of topological semigroups, and that of transformation groups. The former may be found in [5], and the latter in [7]. Semigroup is to mean topological semigroup. If $S$ is a semigroup with identity, 1 , and $N$ is a group in $S$ with 1 in $N$, then $N$ acts as a transformation semigroup on $S$ by left multiplication, and any two distinct orbits of this action are disjoint. Thus, if $M$ is a subset of $S$ which is invariant under this action, we may form the orbit space $M / N$. Whenever we say that $M / N$ is a group, we mean the operation $(N m)\left(N m^{\prime}\right)=N\left(m^{\prime}\right)$ is well-defined, and makes $M / N$ into a group (algebraically speaking).

We denote the multiplicative group of positive reals by $P$, and use $P^{-}$to designate the multiplicative semigroup of nonnegative reals. Referring to [2], for each positive integer $k$, we set

$V_{k}=P \times P \times \cdots \times P$ (k-copies),$\quad V_{k}^{-}=P^{-} \times P^{-} \times \cdots \times P^{-}$(k-copies) and $L_{k}=V_{\mathbf{k}}^{-}-V_{k}$.

We use $e$ to denote the zero of $V_{\boldsymbol{k}}^{-}$, and obtain information about $V_{\boldsymbol{k}}^{-}, V_{k}$, and $L_{k}$ from [2]; for example, $L_{k}$ is a connected ideal in $V_{\boldsymbol{k}}^{-}$.

In what follows, $S$ is to be an abelian semigroup on an $n$-dimensional simply connected manifold with boundary such that the interior of $S$ is a dense, connected, simply connected group, $G$. We do not assume $S$ is compact. Since $G$ is dense in $S$, the identity, 1 , of $G$ is the identity for $S$. We further assume that there is a $k<n+1$ such that $V_{\boldsymbol{k}}^{-} \subset S, 1 \in V_{\boldsymbol{k}}^{-}, G \cap L_{k}=\varnothing$, and $\left(S-G L_{k}\right) / V_{k}$ is a group. It will be shown that $S-G L_{k}=G$.

Received by the editors June 10, 1969.

AMS Subject Classifications. Primary 5480, 2205; Secondary 5478, 2240, 2250.

Key Words and Phrases. Simply connected manifold, boundary, vector semigroup, vector group, dimension, fundamental group, retract.

1 This work is a portion of the author's doctoral dissertation written under the direction of Professor J. G. Horne, at the University of Georgia. 
Since $G$ is the interior of $S, H(1)$ is a Lie group [9]. Since $G$ is dense and open in $S$, it is seen that $G=H(1)$. Further, since $S$ is abelian, $\operatorname{Bd}(S)=S-G$ is an ideal in $S . G$ is a connected, simply connected, $n$-dimensional Lie group, so $G$ is iseomorphic to the $n$-dimensional vector group [4]. If $A$ is a subset of $S, A^{*}$ denotes the closure of $A$.

It is a great pleasure to acknowledge the helpful comments of Professor A. D. Wallace.

Preliminary results. Suppose $V$ is a vector group, and $H$ is a subgroup of $V$ such that $V / H$ is also a vector group. If $\pi: V \rightarrow V / H$ is the natural map, $\pi$ is linear [4], so $H$ is a vector subspace of $V$. This establishes

Lemma 1. If $V$ is a vector group, and $H$ is a subgroup such that $V / H$ is also a vector group, then $H$ is a vector subspace of $V$.

Our first interesting result is

Theorem 1. $G=S-G L_{k}$, and $\operatorname{Bd}(S)=G L_{k}$. Furthermore, $\operatorname{Bd}(S)$ is connected and is an (n-1)-manifold.

Proof. Since $G \cap L_{k}=\varnothing$, and since $\operatorname{Bd}(S)$ is an ideal, $G=S-\operatorname{Bd}(S)$ $\subset S-G L_{k}$. Since $V_{k} \subset H(1)=G, V_{k} \subset S-G L_{k}$. If $t \in S-G L_{k}$, and if $v \in V_{k}$, then $v t \in S-G L_{k}$. For, if $v t \in G L_{k}, t \in\left(v^{-1} G\right) L_{k}=G L_{k}$. We then see that for every $t \in S-G L_{k}, V_{k} t \subset S-G L_{k}$. Since $\left(S-G L_{k}\right) / V_{k}$ is a group and $1 \in V_{k} \subset G \subset S-G L_{k}$, it is seen from [3] that $S-G L_{k}$ $C H(1)=G$. Therefore, $G=S-G L_{k}$.

$G=S-G L_{k}$, so, $\operatorname{Bd}(S)=S-G=G L_{k}$. As mentioned above, $L_{k}$ is connected, so, since $G$ is connected, $\operatorname{Bd}(S)=G L_{k}$ is connected. It then follows $[10]$ that $\operatorname{Bd}(S)$ is an $(\mathrm{n}-1)$-manifold.

We now present

THEOREM 2. Ge $=S e$, and $G e$ is a vector group of dimension not greater than $n-k$. Furthermore, $G_{e}$, the isotropy subgroup of $G$ at $e$ under left multiplication, is connected.

Proof. Since $G$ is iseomorphic to the $n$-dimensional vector group, and since $V_{k}$ is a vector subgroup of $G$, there is a vector group $V_{n-k}$ in $G$ such that $V_{n-k} \times V_{k}$ is iseomorphic to $G$ under $(v, t) \rightarrow v t$. Thus, $G=V_{n-k} V_{k}$, so $G L_{k}=\left(V_{n-k} V_{k}\right) L_{k}=V_{n-k}\left(V_{k} L_{k}\right)$. But, $L_{k}$ is an ideal in $V_{k}^{-}$, so, since $1 \in V_{k}, V_{k} L_{k} \subset L_{k} \subset V_{k} L_{k}$. Thus, $V_{k} L_{k}=L_{k}$, and $G L_{k}=V_{n-k} L_{k}$. From this we see that $(v, t) \rightarrow v t$ maps $V_{n-k} \times V_{\mathbf{k}}^{-}$ homomorphically onto $G \cup G L_{k}=S$.

Since $e$ is the zero of $V_{\boldsymbol{k}}^{-}$, since $G=V_{n-k} V_{k}$, and since $S=V_{n-k} V_{\boldsymbol{k}}^{-}$, we readily see that $G e=V_{n-k} e=S e$. This is the first part of the theorem. 
Now, $e$ is an idempotent in $S$, and there is a one-parameter semigroup in $V_{k}^{-} \subset S$ which has $e$ as its zero. Thus, $S e=G e$ is a deformation retract of $S$. Hence, $G e$ is closed in $S$, so it is locally compact. Also, since $S$ is abelian, $G e$ is algebraically a group with identity $e=1 e$. Therefore [1], $G e$ is a topological group. Furthermore, $v \rightarrow v e$ is a homomorphism from the locally compact, Lindelöf, Hausdorff topological group $V_{n-k}$ onto Ge. Hence, the map is open, and $G e$ is iseomorphic to $\left(V_{n-k}\right) /\left(V_{n-k}\right)_{e}$, where $\left(V_{n-k}\right)_{e}$ is the isotropy subgroup of $V_{n-k}$ at $e$. Since $\left(V_{n-k}\right)_{e}$ is a closed subgroup of $V_{n-k}$, $\left(V_{n-k}\right) /\left(V_{n-k}\right)_{e}$ is iseomorphic to a product of a vector group $V_{p}$ and a toral group $T_{q}$, with $p+q<n-k+1$ [4]. Then, $G e \cong\left(V_{n-k}\right) /\left(V_{n-k}\right)_{e}$ $\cong V_{p} \times T_{q}$.

Letting $\pi_{1}$ denote the fundamental group functor, we have

$$
\begin{aligned}
\pi_{1}(G e) & =\pi_{1}\left(V_{p} \times T_{q}\right)=\pi_{1}\left(V_{p}\right) \oplus \pi_{1}\left(T_{q}\right) \\
& =\pi_{1}\left(T_{q}\right)=Z \oplus Z \oplus \cdots \oplus Z \quad \text { (q-copies). }
\end{aligned}
$$

However, $G e=S e$ is a deformation retract of the simply connected space $S$, so $\pi_{1}(G e)=0$. Therefore, $q=0$, and $G e$ is iseomorphic to $V_{p}, p<n-k+1$. Hence, $G e=S e$ is a vector group of dimension not greater than $n-k$.

$G$ is a vector group, so $G$ is a locally compact, Lindelöf, Hausdorff topological group. Further, $g \rightarrow g e$ is a homomorphism of $G$ onto $G e$, so, as above, $G e$ is iseomorphic to $G / G_{e}$. Hence, $G_{e}$ is a subgroup of the vector group $G$ such that $G / G_{e}$ is a vector group. Hence, by Lemma 1, $G_{e}$ is a vector group, and therefore connected, which concludes the proof of the theorem.

We remark that the proof above can also be used to show that $\left(V_{n-k}\right)_{e}$ is connected.

We now prove a theorem which leads to our characterization theorems. It is

THEOREM 3. The following are equivalent:

(i) $S$ is iseomorphic to $V_{n-k} \times V_{k}^{-}$under the map $(v, t) \rightarrow v t$.

(ii) $v \rightarrow v e$ is an iseomorphism from $V_{n-k}$ onto $G e$.

(iii) $\operatorname{dim} G e=n-k$.

Proof. From the proof of Theorem 2, $(v, t) \rightarrow v t$ is a homomorphism from $V_{n-k} \times V_{k}^{-}$onto $S$, and an iseomorphism from $V_{n-k} \times V_{k}$ onto $G$. Also, $v \rightarrow v e$ is an open homomorphism from $V_{n-k}$ onto $G e$.

( $\Rightarrow$ ii) If $(v, t) \rightarrow v t$ is an iseomorphism, it follows that $v \rightarrow v e$ must be 1-1 from $V_{n-k}$ onto $G e$. Thus, $v \rightarrow v e$ is an iseomorphism from $V_{n-k}$ onto $\mathrm{Ge}$. 
(ii $\Rightarrow$ iii) If $v \rightarrow v e$ is an iseomorphism, then $V_{n-k}$ is homeomorphic to $G e$, so $\operatorname{dim} G e=\operatorname{dim} V_{n-k}=n-k$.

(iii $\Rightarrow$ ii) It is shown in the proof of Theorem 2 that $v \rightarrow v e$ is an open homomorphism from $V_{n-k}$ onto $G e$, and that $G e$ is iseomorphic to $\left(V_{n-k}\right) /\left(V_{n-k}\right)_{e}$. Thus, $n-k=\operatorname{dim} G e=\operatorname{dim}\left[\left(V_{n-k}\right) /\left(V_{n-k}\right)_{e}\right]$. But, [8], $\operatorname{dim}\left[\left(V_{n-k}\right) /\left(V_{n-k}\right)_{e}\right]=\operatorname{dim} V_{n-k}-\operatorname{dim}\left(V_{n-k}\right)_{e}=(n-k)-\operatorname{dim}\left(V_{n-k}\right)_{e}$. Then, $n-k=(n-k)-\operatorname{dim}\left(V_{n-k}\right)_{e}$, so $\operatorname{dim}\left(V_{n-k}\right)_{e}=0$. Hence, $\left(V_{n-k}\right)_{e}$ $=\{1\}$, so $v \rightarrow v e$ is $1-1$, and is thus an iseomorphism from $V_{n-k}$ onto $G e$.

(ii $\Rightarrow$ i) We already know that $(v, l) \rightarrow v l$ is a homomorphism from $V_{n-k} \times V_{\mathbf{k}}^{-}$onto $S$. We now show that it is $1-1$.

Suppose $v, v^{\prime} \in V_{n-k}$ and $t, t^{\prime} \in V_{\boldsymbol{k}}^{-}$such that $v t=v^{\prime} t^{\prime}$. Then, since $e$ is the zero for $V_{\boldsymbol{k}}^{-}, v e=(v t) e=\left(v^{\prime} t^{\prime}\right) e=v^{\prime} e$. Thus, $v=v^{\prime}$, because $s \rightarrow s e$ is an iseomorphism from $V_{n-k}$ onto Ge. Then, $t=v^{-1}(v t)=v^{-1}\left(v^{\prime} t^{\prime}\right)$ $=v^{-1}\left(v t^{\prime}\right)=t^{\prime}$. Hence, $(v, t)=\left(v^{\prime}, t^{\prime}\right)$, and our map is $1-1$.

To show that our map is an iseomorphism, it is now sufficient to show that if $\left\{v_{\rho}\right\}$ and $\left\{t_{\rho}\right\}$ are nets in $V_{n-k}$ and $V_{\boldsymbol{k}}^{-}$respectively such that $v_{\rho} t_{\rho} \rightarrow v t$ for some $v \in V_{n-k}$ and $t \in V_{k}^{-}$, then $\left(v_{\rho}, t_{\rho}\right) \rightarrow(v, t)$. But, if $v_{\rho} t_{\rho} \rightarrow v t$, then $v_{\rho} e=\left(v_{\rho} t_{\rho}\right) e \rightarrow(v t) e=v e$. Since $s \rightarrow s e$ is an iseomorphism, $v_{\rho} \rightarrow v . V_{n-k}$ is a topological group, so $v_{\rho}^{-1} \rightarrow v^{-1}$, and $t_{\rho}=v_{\rho}^{-1}\left(v_{\rho} t_{\rho}\right) \rightarrow v^{-1}(v t)$ $=t$. Hence, $\left(v_{\rho}, t_{\rho}\right) \rightarrow(v, t)$, and we have completed the proof of the theorem.

Principal results. We now give our characterizations of $S$. The first of these is

THEOREM 4. If $k=n, S$ is iseomorphic to $V_{n}^{-}$.

Proof. If $k=n, V_{k}$ is an $n$-dimensional vector subgroup of $G$. Hence, $V_{k}=G$, so $S=G^{*}=\left(V_{k}\right)^{*}=V_{\boldsymbol{k}}^{-}$[2], and we see that $S=V_{\boldsymbol{k}}^{-}$ $=V_{n}^{-}$.

THEOREM 5. If $k=n-1$, and $e$ is not a zero for $S$, then $S$ is iseomorphic to $V_{1} \times V_{n-1}^{-}$.

Proof. $G e=V_{1} e$, so $\operatorname{dim} G e \leqq \operatorname{dim} V_{1}=1$. If $\operatorname{dim} G e=0$, then, since $G e$ is connected and nonempty, $G e=\{e\}$. But, by Theorem 2, $S e=G e=\{e\}$, so $e$ is a zero for $S$. This contradiction implies that $\operatorname{dim} G e=1$, which, by Theorem 3, yields the result.

Theorem 6. If $k=1, S$ is iseomorphic to $V_{n-1} \times V_{1}^{-}$.

Proof. $S$ is an $n$-manifold with boundary, and, by Theorem 1 , $\operatorname{Bd}(S)$ is an $(n-1)$-manifold, and $\operatorname{Bd}(S)=G L_{1}$. Since $L_{1}=\{e\}$, $G L_{1}=G e$. Thus, $\operatorname{dim} G e=n-1$, and the result follows from Theorem 3 .

The last characterization we have obtained to date is 
THEOREM 7. If $k=2, S$ is iseomorphic to $V_{n-2} \times V_{2}^{-}$.

Proof. From Theorem 1, $\operatorname{Bd}(S)=G L_{2}$, and from the proof of Theorem 2, $G=V_{n-2} V_{2}$, and $S=V_{n-2} V_{2}^{-}$. From [2], we may find idempotents $e_{1}$ and $e_{2}$ in $L_{2}$ such that $e_{1} \neq e_{2}, e_{1} \neq e \neq e_{2}, e=e_{1} e_{2}$, and $L_{2}=V_{2}^{-} e_{1} \cup V_{2}^{-} e_{2}$. We then see that

$$
\begin{aligned}
\operatorname{Bd}(S) & =G L_{2}=G\left(V_{2}^{-} e_{1} \cup V_{2}^{-} e_{2}\right) \subset G V_{2}^{-} e_{1} \cup G V_{2}^{-} e_{2} \\
& \subset G L_{2} \cup G L_{2}=G L_{2}=\operatorname{Bd}(S) .
\end{aligned}
$$

Therefore, $\operatorname{Bd}(S)=G V_{2}^{-} e_{1} \cup G V_{2}^{-} e_{2}$. We have, for $i=1,2, G V_{2}^{-} e_{i}=$ $\left(V_{n-2} V_{2}\right) V_{2}^{-} e_{i}=\left(V_{n-2} V_{2}^{-}\right) e_{i}=S e_{i}$. Thus, $\operatorname{Bd}(S)=S e_{1} \cup S e_{2}$. Since each of $e_{1}$ and $e_{2}$ is an idempotent in $S$, each of $S e_{1}$ and $S e_{2}$ is a retract of $S$ and hence closed in $S$, and thus in $\operatorname{Bd}(S)$.

For $i=1$, 2, we see that $S e_{i} \subset\left(G e_{i}\right)^{*}$, because $G^{*}=S$. But, $G \subset S$, so $G e_{i} \subset S e_{i}$. Since $S e_{i}$ is closed, it follows that $\left(G e_{i}\right)^{*}=S e_{i}$. Now, $S e_{i}=\left(V_{n-2} V_{2}^{-}\right) e_{i}=V_{n-2}\left(V_{2}^{-} e_{i}\right)$. However, [2] gives $V_{2}^{-} e_{i}=V_{2} e_{i} \cup\{e\}$, so we have $S e_{i}=V_{n-2}\left[V_{2} e_{i} \cup\{e\}\right] \subset V_{n-2} V_{2} e_{i} \cup V_{n-2} e \subset G e_{i} \cup G e \subset S e_{i}$. Therefore, $S e_{i}=G e_{i} \cup G e$.

If $i \neq j, G e_{i} \cap S e_{j}=\varnothing$. For, if $g \in G$ such that $g e_{i} \in S e_{j}, e_{i} \in S e_{j}$, and there is an $s \in S$ such that $e_{i}=s e_{j}$. Then, $e_{i}=e_{i}^{2}=\left(s e_{j}\right) e_{i}=s e$, so, $e=e_{i} e_{j}=(s e) e_{j}=s e=e_{i}$, which is a contradiction. Therefore, $G e_{i} \cap S e_{j}=\varnothing$. Since $G e \subset S e_{j}$, it follows that for each $i=1,2 G e_{i} \cap G e$ $=\varnothing$. Hence, $S e_{i}-G e_{i}=G e$.

From above, $\operatorname{Bd}(S)=S e_{1} \cup S e_{2}=G e_{1} \cup G e \cup G e_{2}$, and no two of these intersect. Thus, $\operatorname{Bd}(S)-G e_{1}=G e \cup G e_{2}=S e_{2} . S e_{2}$ is closed in $\operatorname{Bd}(S)$, so $G e_{1}$ is open in $\mathrm{Bd}(S)$. Letting $\partial\left(G e_{1}\right)$ be the boundary of $G e_{1}$ in $\operatorname{Bd}(S)$, we see, since $G e_{1}$ is open in $\operatorname{Bd}(S)$, that $\partial\left(G e_{1}\right)=\left(G e_{1}\right)^{*}-G e_{1}$ $=S e_{1}-G e_{1}=G e$. Furthermore, $e_{2} \notin\left(G e_{1}\right)^{*}=S e_{1}$ from above, so we see that $G e_{1}$ is a nonempty, nondense, open subset of the $(n-1)$-manifold $\operatorname{Bd}(S)$. Hence [6], $\operatorname{dim} G e=\operatorname{dim} \partial\left(G e_{1}\right)=(n-1)-1=n-2$. The theorem follows from Theorem 3.

ConcLusion. The results of this paper give a partial characterization of $S$. It should be observed that in all cases presented, $S$ cannot be compact. One wonders whether or not there is a compact $S$ which satisfies our criterion. One also queries whether or not $S$ is iseomorphic to $V_{n-k} \times V_{\mathbf{k}}^{-}$for all $k$. We are presently working on these problems.

\section{BIBLIOGRAPHY}

1. Robert Ellis, $A$ note on the continuity of the inverse, Proc. Amer. Math. Soc. 8 (1957), 372-373. MR 18, 745.

2. T. H. McH. Hanson, Actions that fiber and vector semigroups, Trans. Amer. Math. Soc. (to appear). 
3. - Concerning vector semigroups, Dissertation, Univ. of Georgia, Athens, 1968.

4. G. Hochschild, The structure of Lie groups, Holden-Day, San Francisco Calif., 1965. MR 34 \#7696.

5. K. H. Hofmann and P. S. Mostert, Elements of compact semigroups, Merrill, Columbus, Ohio, 1966.

6. W. Hurewicz and H. Wallman, Dimension theory, Princeton Math. Series, vol. 4, Princeton Univ. Press, Princeton, N.J., 1941. MR 3, 312.

7. D. Montgomery and L. Zippin, Topological transformation groups, Interscience, New York, 1955. MR 17, 383.

8. P. S. Mostert, Sections in principal fibre spaces, Duke Math, J. 23 (1956), 57-71. MR 17, 771.

9. P. S. Mostert and A. L. Shields, Semigroups with identity on a manifold, Trans. Amer. Math. Soc. 91 (1959), 380-389. MR 21 \#4204.

10. P. M. Rice, A course in algebraic topology, Notes, Univ. of Georgia, Athens, 1965-66.

University of Georgia, Athens, Georgia 30601

University of Florida, Gainesville, Florida 32601 